\title{
A NOTE ON ONE-DIMENSIONAL ATTRACTING SETS IN THE THREE-SPHERE
}

\author{
JOEL C. GIBBONS ${ }^{1}$
}

\begin{abstract}
This paper is an application of Williams' results for one-dimensional attracting sets to the three-sphere. Our objective is to classify up to $\Omega$-conjugacy all diffeomorphisms of $S^{3}$ satisfying Smale's axioms $\mathrm{A}$ and $\mathrm{B}$ and the condition that the nonwandering set consists of zero- and one-dimensional sinks and sources.
\end{abstract}

Throughout this paper we will rely repeatedly on the following facts about a sink $\Lambda, \operatorname{dim} \Lambda=r$, of an Anosov-Smale diffeomorphism $f$ of a manifold $M^{n}$. They are a special case of $[3, \S 4]$, and are equivalent to the claims in $[1, \S 1.3]$.

$\Lambda$ has an open neighborhood $N$, called fundamental, such that

(1) $\bigcap_{k=0}^{\infty} f^{k}(N)=\Lambda, \bigcap_{k=0}^{\infty} f^{-k}(N)=W^{s}(\Lambda)$,

(2) bd $N$ has a tubular neighborhood $V$ such that $V \cap \Omega(f)=\varnothing$,

(3) $f^{k+1}(N) \subset f^{k}(N)$ for all $k$,

(4) $N$ has a foliation $\mathscr{G} N$ by smooth $(n-r)$-cells, $G$, transverse to $\Lambda$,

(5) $K=N / \mathscr{G} N$ is a smooth branched $r$-manifold, and

(6) if $p: N \rightarrow K$ is the quotient, $p$ is a homotopy equivalence.

LEMMA 1. Let $M^{n}$ be a compact, connected, oriented manifold and let $f$ be an Anosov-Smale diffeomorphism of $M$. If $\Omega(f)$ consists of sinks $\Lambda_{1}$, $\Lambda_{2}, \cdots, \Lambda_{s}$ and sources $\Lambda_{s+1}, \cdots, \Lambda_{t}$, and codim $\Lambda_{j} \geqq 2$, for all $j=$ $1, \cdots, t$, then $s=1$ and $t=2$, and $\Lambda_{1}$ and $\Lambda_{2}$ are connected.

Proof. We claim first that if $\Lambda$ is any sink, it has at most finitely many components. If not, $\operatorname{Per}(f / \Lambda)$ cannot be contained in any finite set of components, since it is dense in $\Lambda$. Every component which contains a periodic point is a basic attractor of some iterate of $f$, and therefore has a neighborhood disjoint from the other components. In this way construct an open cover of $M$ with no finite subcover.

Let $A=\bigcup_{j=1}^{s} \Lambda_{j}$ and $A^{*}=\Omega(f)-A$. We claim that $M-A$ is connected.

Presented to the Society, September 2, 1971 under the title One-dimensional basic sets in the three-sphere; received by the editors October 16, 1970.

AMS 1970 subject classifications. Primary 58F15; Secondary 55J30, 57D30.

Key words and phrases. Generalized solenoid, Alexander duality.

${ }^{1}$ Supported by NSF grant GP 19815.

(C) American Mathematical Society 1972 
By Alexander duality [4, p. 296] and the exact homology sequence of a pair

$$
\breve{H}^{n-1}(A) \cong H_{1}(M, M-A) \cong \bar{H}_{0}(M-A),
$$

where $\bar{H}_{*}$ is augmented homology, and $\breve{H}^{*}$ of a closed set is the direct limit of singular cohomology over a cofinal family of open neighborhoods. An easy computation using fundamental neighborhoods and fact (6) shows that the left side is trivial.

Take a sufficiently high iterate of $f$ so that all components of $A^{*}$ are basic sets. By fact (1), applied to $f^{-1}$, the unstable neighborhood of each component is open in $M-A$. The same argument applies to $A$.

LeMma 2. Let $f$ be a north pole-south pole map and let $\Lambda$ be its sink. If $\operatorname{dim} \Lambda=1, \Lambda, f \mid \Lambda$ is a generalized solenoid. If $N$ is a fundamental neighborhood and $(K, g)$ is a presentation, $f_{*}: H_{1}(N) \rightarrow H_{1}(N)$ is conjugate via $p_{*}$ to $g_{*}: H_{1}(K) \rightarrow H_{1}(K) . f_{*}$ is not nilpotent.

Proof. The first claim is a special case of [1, Theorem D]. In the course of the proof, Williams proves that

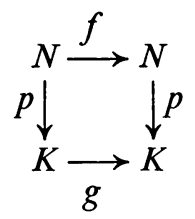

commutes, from which the second claim follows.

To prove the last claim, we can assume that $K$ is orientable, or else we take the double covering. Then, Williams shows [2, Theorem E] that, for some positive integer $k,\left(K, g^{k}\right)$ is shift equivalent to $(\bar{K}, \bar{g})$, where $\bar{K}$ is elementary. Since $\bar{g}$ is an immersion, no iterate of $\bar{g}_{*}$ has rank zero. Shift equivalence preserves rank, so the claim follows.

We are ready to prove the classification, which is

THEOREM. If $f$ is a north pole-south pole map, $\Omega(f)$ consists of two connected basic sets, $\Lambda_{1}$ and $\Lambda_{2}$, a sink, and a source, resp., and

(a) $\operatorname{dim} \Lambda_{1}=\operatorname{dim} \Lambda_{2}=\operatorname{dim} \Omega(f)$,

(b) if $\operatorname{dim} \Omega(f)=0, \Omega(f)$ consists of two fixed points, and

(c) if $\operatorname{dim} \Omega(f)=1, \Lambda_{1}, f / \Lambda_{1}$ and $\Lambda_{2}, f^{-1} / \Lambda_{2}$ are generalized solenoids. If $\left(K_{1}, g_{1}\right)$ and $\left(K_{2}, g_{2}\right)$ are presentations, resp., $H_{1}\left(K_{1}\right)$ and $H_{1}\left(K_{2}\right)$ are isomorphic free groups, and under the isomorphism $g_{1^{*}}=\left(g_{2^{*}}\right)^{t}$.

Proof. The first assertion follows from Lemma 1. Let $N$ be a fundamental neighborhood of $\Lambda_{1} ; M=\operatorname{int}\left(S^{3}-N\right)$ is a fundamental neighborhood of $\Lambda_{2}$. Let $N_{k}=f^{k}(N)$ and $M_{k}=f^{k}(M)$. By Alexander duality and 
the exact homology sequence of a pair, for all integers $k$,

(i) $\breve{H}^{1}\left(\mathrm{cl} N_{k}\right) \cong H_{2}\left(S^{3}, M_{k}\right) \cong H_{1}\left(M_{k}\right)$.

Since bd $N$ has a tubular neighborhood in $S^{3}$, by $[4$, p. 290] and standard arguments,

(ii) $\check{H}^{1}\left(\mathrm{cl} N_{k}\right) \cong H^{1}\left(\mathrm{cl} N_{k}\right) \cong H^{1}\left(N_{k}\right)$.

$H^{1}\left(N_{k}\right)$ is free finitely generated, by fact (6), so by the universal coefficient theorem for cohomology $[4$, p. 248],

(iii) $H_{1}\left(N_{k}\right) \cong \operatorname{Hom}\left(H^{1}\left(N_{k}\right) ; Z\right)$.

All these isomorphisms are natural. Together they imply that

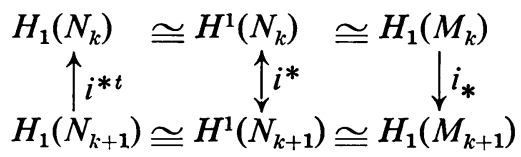

commutes, where $i$ is the inclusion. By naturality $i^{* t}=i_{*}$. Claims (a) and (b) follows from observing that, if $\operatorname{dim} \Lambda_{1}=0, N_{k}$ is contractible, so $\left(f^{-1} / M\right)_{*}$ is necessarily nilpotent. Then, from Lemma 2 we know $\operatorname{dim} \Lambda_{2}=0$.

The last claim requires a computation. We will show that if $\left(f / N_{k}\right)_{*}: H_{1}\left(N_{k}\right) \rightarrow H_{1}\left(N_{k}\right)$ and $\left(f^{-1} / M_{k+1}\right): H_{1}\left(M_{k+1}\right) \rightarrow H_{1}\left(M_{k+1}\right),\left(f / N_{k}\right)_{*}=$ $\left(f^{-1} / M_{k+1}\right)_{*}^{t}$. Choose bases for the groups in the above diagram for which the matrix representations of these maps are equal, respectively, to those of $i_{*}: H_{1}\left(N_{k+1}\right) \rightarrow H_{1}\left(N_{k}\right)$ and $i_{*}: H_{1}\left(M_{k}\right) \rightarrow H_{1}\left(M_{k+1}\right)$. The claim follows from the diagram. These matrices are invariant of $k$, so we are done.

\section{BIBLIOGRAPHY}

1. R. F. Williams, One-dimensional non-wandering sets, Topology 6 (1967), 473-487. MR 36 \#897.

2. - Classification of one-dimensional attractors, Northwestern University, Evanston, Ill. (mimeographed notes).

3. M. Hirsch et al., Neighborhoods of hyperbolic sets, Invent. Math. 9 (1969/70), 121-134. MR 41 \#7232.

4. E. Spanier, Algebraic topology, McGraw-Hill, New York, 1966. MR 35 \#1007.

Department of Mathematics, Northwestern University, Evanston, Illinois 60201

Current address: Department of Mathematics, Chicago State College, Chicago, Illinois 60621 